\title{
THE CUPID CODE DEVELOPMENT AND ASSESSMENT STRATEGY
}

\author{
J.J. JEONG*, H.Y. YOON, I.K. PARK, and H.K. CHO \\ Korea Atomic Energy Research Institute (KAERI) \\ 1045 Daeduk-daero, Yuseong-gu, Daejeon 305-353, South Korea \\ *Corresponding author. E-mail : jjjeong@kaeri.re.kr
}

Received December 06, 2010

A thermal-hydraulic code, named CUPID, has been being developed for the realistic analysis of transient two-phase flows in nuclear reactor components. The CUPID code development was motivated from very practical needs, including the analyses of a downcomer boiling, a two-phase flow mixing in a pool, and a two-phase flow in a direct vessel injection system. The CUPID code adopts a two-fluid, three-field model for two-phase flows, and the governing equations are solved over unstructured grids with a semi-implicit two-step method. This paper presents an overview of the CUPID code development and assessment strategy. It also presents the code couplings with a system code, MARS, and, a three-dimensional reactor kinetics code, MASTER.

KEYWORDS : The CUPID Code Development, Assessment Strategy, Component Scale, Two-Fluid Three-Field Model, Code Couplings

\section{INTRODUCTION}

State-of-the-art thermal-hydraulic system codes have reached a high degree of maturity for the purpose of analyzing a wide range of accident conditions in light water reactors. Nevertheless, there still remain some shortcomings in these codes, which are mainly related to limitations in physical modeling approach or numerical solution methods $[1,2]$. These limitations includes, in particular, one-dimensional models for multi-dimensional flow phenomena, flow regime maps that were developed from steady-state, fully-developed flow conditions, robust but highly diffusive numerical methods, e.g., first-order approximations for time and space discretization, etc. These limitations demand a new attempt at the development of improved thermal-hydraulic codes.

Rapid advances in computing power have facilitated the use of computational fluid dynamics (CFD) codes for nuclear reactor safety. Accordingly, the OECD Committee on the Safety of Nuclear Installations has identified the type of the nuclear reactor safety problems, where extending CFD to two-phase flow may bring a real benefit [3]. These problems involve dry-out, departure from nucleate boiling, pressurized thermal shock, pool heat exchanger, and so on. However, since the two-phase flow models in CFD codes are not as mature as those in the one-dimensional system codes or single-phase flow models of CFD codes, a lot of further developments are still needed for these applications. Basically, two-phase flow involves various phenomena at different time and spatial scales. Physical models for some two-phase flow regimes are still not available and, furthermore, a smaller scale calculation requires huge computational resource that might be currently not attainable. Thus, universal two-phase computation tools seem unlikely to be available for a few decades. This possibility has fostered a multiscale approach to two-phase flows; that refers to the combined use of macroscale, mesoscale and microscale computation tools with feedback between different scale codes [4]. In general, time-averaged multi-fluid models using the Reynolds Average Navier-Stokes (RANS) approach are adopted for the macroscale and mesoscale, whereas a pseudo direct numerical simulation (DNS) with an interface tracking method is mainly used at the microscale.

Various efforts have been being made, in separate or systematic ways, to realize the multiscale approach for industrial use. The ASTAR project [5], which started in 2001, identified the current status and future perspectives in the field of advanced numerical simulation of threedimensional two-phase flow processes. The NEPTUNE project was launched in 2001 [6,7], based on industrial needs of two-phase flow analysis. It aims at preparing a new generation of two-phase flow thermal-hydraulics tools, covering the whole range of modeling scales and allowing easy multiscale and multidisciplinary calculations. 
Significant progress has been already established in the system-scale, component-scale, mesoscale and microscale. The NEPTUNE-CFD and TRIO-U codes are two-phase codes at mesoscale and microscale, respectively. The MSMA project focuses on multiscale modeling and analysis of convective boiling, which ultimately aims at the predictive capability of critical heat flux [8]. The ACE-3D code, which can be categorized into a mesoscale code, adopts an advanced model by combining a twofluid model and an interface tracking method [9]. The two-fluid model is used to model small interface structure, such as bubbles or droplets that are smaller than the computational cells. The interface tracking method is used for large interface structure, such as liquid film or large bubbles. All these approaches still have many challenges for application to full range of two-phase flow regimes.

The Korea Atomic Energy Research Institute has been developing a three-dimensional thermal-hydraulic code, called CUPID, which was motivated from practical needs for the realistic simulation of two-phase flows in nuclear reactor components $[10,11]$. The need for a multi-dimensional analysis is further increasing with advanced design features, such as a direct vessel injection (DVI) system, a gravity-driven safety injection system, and a passive secondary cooling system. Some phenomena in these systems are characterized by multi-dimensional flow behavior with weak driving force. For example, downcomer boiling with the DVI system [12] and twophase flow mixing in a pool required a three-dimensional analysis with enhanced accuracy to reproduce the natural circulations accelerated by two-phase flow. However, neither the three-dimensional component of a system code nor some commercial CFD codes led to practical results because of the complicated multi-dimensional nature with dramatic phase changes. The CUPID code development was initiated to overcome these drawbacks.

The CUPID code is a macroscale code and it can be a stepping stone to a finer-scale code in the future. The primary objective of the CUPID code development is to establish a standalone code for two-phase flow in nuclear reactor components. The code is then to be coupled with a system code to take advantage of the two codes, and also with a reactor kinetics code for a coupled analysis of transient nuclear reactor core. In addition to these practical objectives, the CUPID code aims at explorations into the current limitations of two-phase flow modeling for refined calculations.

The CUPID code adopts a two-fluid, three-field model for two-phase flows. It is a straightforward extension of the two-fluid single pressure model [13], in which the local instant formulation of a two-phase flow is time-averaged. During the averaging process, the local two-phase interface structure is lost and, instead, the presence of the interface is considered by using a model, i.e., a flow regime map. This situation results in substantial problems for two-phase flow simulation under a variety of flow conditions. These problems will be specifically identified during the code development. The CUPID code is designed to have features for both open and porous media. In two-phase momentum equations, nondrag forces, such as lift, wall lubrication, and turbulent dispersion forces, are modeled in addition to the interfacial drag forces and these non-drag forces are selectively activated. The features distinguish the CUPID code from the three-dimensional components of system codes, such as RELAP5-3D [14], MARS [15], and CATHARE 2 [16]. There are, however, a lot of limitations in physical models of the open media approach for some flow regimes. Thus, the applications of CUPID are restricted to certain flow regimes, and the assessment strategy should be established in this context.

This paper presents an overview of the CUPID code development status and assessment strategy. In Section 2, we present physical models of two-phase flows as well as a heat conduction model. In Section 3, we discuss the numerical solution methods for the flow model and highlight some improved features. In Section 4, we describe the code couplings with a system code and a reactor kinetics code. In the final section, we present the strategy and status of the code verification and validation.

\section{PHYSICAL MODELS FOR TWO-PHASE FLOWS}

The CUPID code is basically a macroscale code, of which physical models are based on porous media approach. However, some features for open media analysis were also implemented for optional use. This section presents the basic governing equations and physical models for open media analysis.

\subsection{Basic Governing Equations}

To describe two-phase flows, a transient two-fluid three-field model is adopted. The three fields include a continuous liquid, an entrained liquid, and a vapor field $[15,17]$. The three-field formulation of a two-phase flow is a straightforward extension of the two-fluid single pressure model [13], in which the local instant formulation of a two-phase flow is time-averaged. The mass, energy, and momentum equation for each fields are established separately and, then, they are linked by the interfacial mass, energy, and momentum transfer models.

The continuity equation for $\mathrm{k}$-field is

$$
\frac{\partial}{\partial t}\left(\alpha_{k} \rho_{k}\right)+\nabla \cdot\left(\alpha_{k} \rho_{k} \underline{u}_{k}\right)=\Omega_{k},
$$


The noncondensable gases, when present, are contained in the vapor field, and these are assumed to move with the same velocity, and have the same temperature as the vapor phase. The continuity equation for the total noncondensable component is given as

$$
\frac{\partial}{\partial t}\left(\alpha_{g} \rho_{g} X_{n}\right)+\nabla \cdot\left(\alpha_{g} \rho_{g} X_{n} \underline{u}_{g}\right)=0,
$$

where the noncondensable gas quality $X_{n}$ is defined as the ratio of the noncondensable gas mass to the total gaseous phase mass.

The momentum equation for $\mathrm{k}$-field is:

$$
\begin{aligned}
& \frac{\partial}{\partial t}\left(\alpha_{k} \rho_{k} \underline{u}_{k}\right)+\nabla \cdot\left(\alpha_{k} \rho_{k} \underline{u}_{k} \underline{u}_{k}\right)=-\alpha_{k} \nabla P \\
& +\nabla \cdot\left[\alpha_{k}\left(\tau_{k}+\tau_{k}^{T}\right)\right]+\alpha_{k} \rho_{k} \underline{g}+\underline{F}_{i k}+\underline{F}_{k}^{N D},
\end{aligned}
$$

where $F_{i k}$ is the interfacial momentum transfer due to interfacial drag, virtual mass, and interfacial mass transfer. $\underline{F}_{k}^{N D}$ is the interfacial momentum transfer terms due to non-drag forces. The turbulent Reynolds stress is obtained by using the standard k- $\varepsilon$ model or the mixing length model.

It is assumed that the continuous liquid and the entrained liquid are in a thermal equilibrium, i.e., $T_{d}=T_{l}$. As a result, only two energy equations are used, i.e., one for the vapor field and one for the combined liquid field:

$$
\begin{gathered}
\frac{\partial}{\partial t}\left(\alpha_{g} \rho_{g} e_{g}\right)+\nabla \cdot\left(\alpha_{g} \rho_{g} e_{g} \underline{u}_{g}\right)=E_{g}^{D} \\
-P \frac{\partial}{\partial t} \alpha_{g}-P \nabla \cdot\left(\alpha_{g} \underline{u}_{g}\right)+Q_{i g}-Q_{g l}, \\
\frac{\partial}{\partial t}\left[\left(1-\alpha_{g}\right) \rho_{l} e_{l}\right]+\nabla \cdot\left[\left(\alpha_{l} \underline{u}_{l}+\alpha_{d} \underline{u}_{d}\right) \rho_{l} e_{l}\right]=E_{l}^{D} \\
-P \frac{\partial}{\partial t}\left(1-\alpha_{g}\right)-P \nabla \cdot\left(\alpha_{l} \underline{u}_{l}+\alpha_{d} \underline{u}_{d}\right)+Q_{i l}+Q_{g l}
\end{gathered}
$$

where $E_{k}^{D}$ includes the k-phase conduction, turbulent energy source, and viscous dissipation.

For mathematical closure, the undefined terms and coefficients in the right-hand side of the governing equations should be established. These are presented in the remainder of Section 2. Equations of the states are also needed. In the CUPID code, the phasic density and temperature of the liquid field are expressed as functions of the pressure and internal energy:

$$
\rho_{l}=\rho_{l}\left(P, e_{l}\right),
$$

$$
T_{l}=T_{l}\left(P, e_{l}\right) .
$$

The properties for the gaseous phase are calculated assuming a modified Gibbs-Dalton mixture of steam and an ideal noncondensable gas:

$$
\rho_{g}=\rho_{g}\left(P, e_{g}, X_{n}\right)
$$

$$
T_{g}=T_{g}\left(P, e_{g}, X_{n}\right)
$$

$$
P_{s}=P_{s}\left(P, e_{g}, X_{n}\right)
$$

The saturation property is represented as a function of the pressure:

$$
T_{\text {sat }}=T_{\text {sat }}\left(P_{s}\right) .
$$

\subsection{Local Two-Phase Flow Regime and Interfacial Transfer Terms}

In a two-phase flow, the interfacial area and interfacial transfer depend on the shape of the interface. However, during the averaging process of the two-fluid formulation, the local interface structure is lost. Thus, the conventional system-scale and component-scale codes use flow regime maps to define the topology of the interface.

\subsubsection{Local Two-Phase Flow Regime}

The traditional flow regime concept, however, is not applicable to open medium analyses because, in the traditional concept, every cell is supposed to contain a wall boundary for the cross sectional average of a flow parameter. To address this issue, CFD-BWR code [18], built on the foundation of STAR-CD [19], proposed an inter-phase surface topology map recently. Their works have attempted simulations of not only dispersed flows but also flows involving a local sharp interface such as slug and annular mist flows. According to their approach, 
the three main types of local inter-phase surface topologies and the transitional topologies are distinguished. As shown in Fig. 1, three main topologies are a bubbly flow topology, a mist flow topology and a sharp interface topology. The transitional topologies are the overlapping regions of two or three main topologies [18]. Tentner et al. [20, 21], Ioilev et al. [22] and Ustinenko et al. [23] have established significant progress to verify and validate this approach.

The inter-phase topology concept proposed by Tentner et al. was employed in the CUPID code. The topology in each mesh cell is determined by two parameters, a void fraction and a void fraction difference function $\gamma$, which is expressed as

$$
\gamma=0.5 \cdot \vec{\delta} \cdot\left[\left|\frac{\partial \alpha_{g}}{\partial x}\right| \mathbf{i}+\left|\frac{\partial \alpha_{g}}{\partial y}\right| \mathbf{j}+\left|\frac{\partial \alpha_{g}}{\partial z}\right| \mathbf{k}\right]
$$

where

$$
\vec{\delta}=\frac{V_{i}}{\sum_{f}\left|S_{f, x}\right| / N_{f}} \mathbf{i}+\frac{V_{i}}{\sum_{f}\left|S_{f, y}\right| / N_{f}} \mathbf{j}+\frac{V_{i}}{\sum_{f}\left|S_{f, z}\right| / N_{f}} \mathbf{k}
$$

The parameters for topology transition criteria in Fig. 1 are $\alpha_{g, b c}=0.3, \alpha_{g, c m}=0.95, \gamma_{1}=0.2$, and $\gamma_{2}=0.4$.

The definition of $\gamma$ and the criterion of the topology change from the transition region to the mist were modified from those in CFD-BWR in order to apply this method to unstructured meshes and to avoid numerical instability, respectively. Once the local inter-phase topology is determined for each cell, the interfacial area and interface transfer models, thereafter, are defined depending on the topology of each cell.

\subsubsection{Interfacial Area Concentration}

The following interfacial area concentration models are adopted in the CUPID code.

For the bubble topology [24]:

$$
a_{i}=\left(\pi D_{b}^{2}\right)\left(\frac{6 \alpha_{g}}{\pi D_{b}^{3}}\right)=\frac{6 \alpha_{g}}{D_{b}},
$$

$$
D_{b}\left(P, \alpha_{g}\right)=10.06\left(\frac{10^{5}}{P}\right)^{0.098} \sqrt{\frac{\sigma}{9.8\left(\rho_{l}-\rho_{g}\right)}} \cdot\left[\min \left(\alpha_{g}, 0.118\right)\right]^{0.35} .
$$
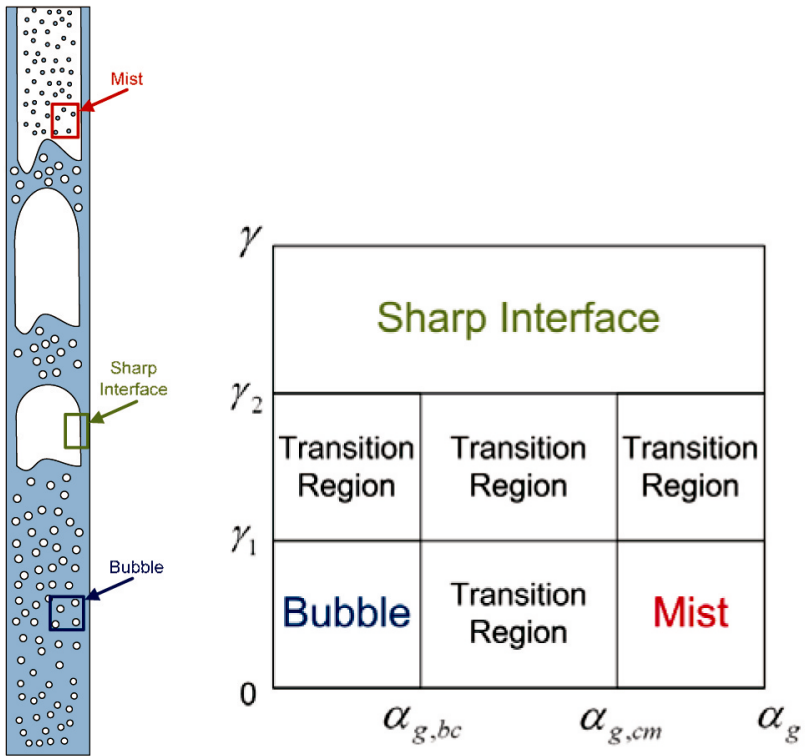

Fig. 1. Inter-Phase Surface Topology Concept

For the mist topology [25]:

$$
\begin{gathered}
a_{i}=\frac{6\left(1-\alpha_{g}\right)}{D_{d}}, \\
D_{d}\left(P, \alpha_{g}\right)=0.01 \frac{\sigma}{\rho_{g} j_{g}^{2}} \operatorname{Re}_{g}^{2 / 3}\left(\frac{\rho_{g}}{\rho_{f}}\right)^{-1 / 3}\left(\frac{\mu_{g}}{\mu_{f}}\right)^{2 / 3} .
\end{gathered}
$$

For the sharp interface topology [26]:

$$
a_{i}=\left|\nabla \alpha_{g}\right|
$$

However, in this approach, only spherical shape bubble, drop and smooth interface are considered. In order to simulate a two-phase flow more precisely where distorted bubbles or wavy interface appear, it is necessary to implement additional models. Currently, the interfacial terms for transition topologies are obtained by an interpolation.

\subsubsection{Interfacial Momentum Transfer}

The interfacial momentum transfer term, $\underline{F}_{i k}$ in Eq. 
(3), includes the interfacial drag, the virtual mass force, and the momentum exchange due to the interface mass transfer. For simplicity, the virtual mass term is introduced later. Then, $F_{i k}$ is written as follows:

$$
\begin{gathered}
\underline{F}_{i g}=\underline{F}_{g l}+\underline{F}_{g d}+\Gamma_{g} \underline{u}_{g i}, \\
\underline{F}_{i l}=\underline{F}_{g l}-(1-\eta) \Gamma_{g} \underline{u}_{l i}-S_{E} \underline{u}_{l}+S_{D E} \underline{u}_{d}, \\
\underline{F}_{i d}=\underline{F}_{g d}-\eta \Gamma_{g} \underline{u}_{d i}+S_{E} \underline{u}_{l}-S_{D E} \underline{u}_{d} .
\end{gathered}
$$

The interface velocities, $\underline{u}_{k i}$, are needed to obtain the interfacial momentum transfer due to the interface mass transfer. These are determined using a donor formulation concept [14]. The interfacial drag force terms in the CUPID code are listed in Table 1. As for the entrainment $S_{E}$ and de-entrainment rates $S_{D E}$, the conventional models based on a one-dimensional approach are currently used, which should be improved later.

The virtual mass force model developed by Drew et al. [27] was applied:

$$
\underline{F}_{v m, g}=C_{g}^{v m} \alpha_{g} \alpha_{l} \rho_{m} \frac{\partial\left(\underline{u}_{l}-\underline{u}_{g}\right)}{\partial t} .
$$

where

$$
\begin{aligned}
& \rho_{m}=\left(\alpha_{g} \rho_{g}+\alpha_{l} \rho_{l}\right), \\
& C_{g}^{v m}=\left\{\begin{array}{l}
\frac{\left(1+2 \alpha_{g}\right)}{2\left(1-\alpha_{g}\right)} \text { for } \alpha_{g}<0.5, \\
\frac{\left(3-2 \alpha_{g}\right)}{2 \alpha_{g}} \text { for } 0.5 \leq \alpha_{g}<1 .
\end{array}\right.
\end{aligned}
$$

\begin{tabular}{|c|c|}
\hline Local flow regime & Interfacial drag force \\
\hline Bubble & $\begin{array}{c}\underline{F}_{g l}=\frac{1}{8} a_{i} \rho_{c} C_{D}\left|\underline{u}_{g}-\underline{u}_{l}\right|\left(\underline{u}_{g}-\underline{u}_{l}\right) \\
\text { where } C_{D}=\left\{\begin{array}{l}\frac{24}{\operatorname{Re}_{b}}\left(1+0.15 \mathrm{Re}_{b}^{0.687}\right) \text { for } 0<\mathrm{Re}_{b} \leq 1000 \\
0.44 \text { for } \operatorname{Re}_{b}>1000\end{array}\right.\end{array}$ \\
\hline Mist & $\begin{array}{l}\underline{F}_{g d}=\frac{1}{8} a_{i} \rho_{c} C_{D}\left|\underline{u}_{g}-\underline{u}_{d}\right|\left(\underline{u}_{g}-\underline{u}_{d}\right) \\
\text { where } C_{D} \text { is obtained for droplets. }\end{array}$ \\
\hline Sharp interface & $\begin{array}{c}\underline{F}_{g l}=\frac{1}{2} a_{i} \rho_{g} C_{i}(\phi)\left|\underline{u}_{g}-\underline{u}_{l}\right|\left(\underline{u}_{g}-\underline{u}_{l}\right) \\
\text { where } C_{i}(\varphi)=C_{i, \text { tan }}+\left(C_{i, o r t}-C_{i, \text { tan }}\right)|\cos \varphi|, C_{i, \text { tan }}=0.005, C_{i, o r t}=1, \text { and } \\
\cos \varphi=\frac{\left(\underline{u}_{g}-\underline{u}_{l}\right) \cdot \nabla \alpha_{g}}{\left|\left(\underline{u}_{g}-\underline{u}_{l}\right)\right|\left|\nabla \alpha_{g}\right|} .\end{array}$ \\
\hline Transition & $\begin{array}{c}\underline{F}_{g l}=\chi_{i}\left(\underline{u}_{g}-\underline{u}_{l}\right) \\
\text { where }\left(\chi_{i}\right)_{\text {transition }}=w \cdot\left(\chi_{i}\right)_{1}+(1-w) \cdot\left(\chi_{i}\right)_{2}, \\
w=\frac{\alpha_{g, c m}-\alpha_{g}}{\alpha_{g, c m}-\alpha_{g, b c}} \text { or } w=\frac{\gamma_{2}-\gamma}{\gamma_{2}-\gamma_{1}}\end{array}$ \\
\hline
\end{tabular}

It is noted that the virtual mass force was applied for all topologies and, however, the other non-drag forces

Table 1. The Interfacial Drag Force Models in the CUPID Code 
were considered only when the bubble topology was identified.

The non-drag force term, $\underline{F}_{k}^{N D}$ in Eq. (3), represents the forces acting perpendicular to the mean flow direction, including the lift, wall lubrication, and turbulent dispersion forces [10].

$$
\underline{F}_{k}^{N D}=\underline{F}_{l i f t, k}+\underline{F}_{w l, k}+\underline{F}_{t d, k} .
$$

The lift force is given in terms for the slip velocity and the curl of the continuous phase velocity by:

$$
\underline{F}_{l i f t, g}=-\alpha_{g} \rho_{l} C_{L}\left(\underline{u}_{g}-\underline{u}_{l}\right) \otimes\left(\nabla \otimes \underline{u}_{l}\right) .
$$

The lift force coefficient suggested by Tomiyama [28] is applied:

$C_{L}=\mid \begin{aligned} & \min \left[0.288 \tanh \left(0.121 \mathrm{Re}_{b}\right), f\left(E_{o, H}\right)\right], \text { if } E_{o, H}<4 \\ & f\left(E_{o, H}\right), \text { if } 4 \leq E_{o, H}<10 \\ & -0.27, \quad \text { if } 10 \leq E_{o, H}\end{aligned}$,

where

$$
\begin{aligned}
\operatorname{Re}_{b} & =\frac{\rho_{l}\left|\underline{u}_{g}-\underline{u}_{l}\right| D_{b}}{\mu_{l}}, \\
f & =0.00105 E_{o, H}^{3}-0.0159 E_{o, H}^{2}-0.0204 E_{o, H}+0.474, \\
E_{o, H} & =\frac{g\left(\rho_{l}-\rho_{g}\right) d_{H}^{2}}{\sigma}, \\
d_{H} & =D_{b}\left(1+0.163 E_{o}^{0.757}\right)^{1 / 3}, \\
E_{o} & =\frac{g\left(\rho_{l}-\rho_{g}\right) D_{b}^{2}}{\sigma} .
\end{aligned}
$$

For the wall lubrication force, the Antal et al.'s model [29] is used:

$$
\underline{F}_{w l, g}=\frac{\alpha_{g} \rho_{l} C_{w l}\left|\underline{u}_{g}-\underline{u}_{l}\right|^{2}}{D_{b}} \max \left(0, C_{1}+C_{2} \frac{D_{b}}{y_{w}}\right) \vec{n}_{w},
$$

where $C_{1}=-0.01$ and $C_{2}=-0.05 . \vec{n}_{w}$ is the surface normal vector of the wall. The wall lubrication force is limited within $y_{w}<5 D_{b}$. The turbulent dispersion force is the result of the turbulence fluctuations of the liquid velocity. Lahey et al. [30] derived an equation for the force per unit volume:

$$
\underline{F}_{t d, g}=C_{T D} \rho_{l} k_{l} \nabla \alpha_{l}
$$

where the coefficient $C_{T D}$ is set to 0.1 . A more generalized non-uniform turbulent dispersion coefficient on the basis of homogeneous turbulence was proposed by de Bertodano [31], which depends on the Stokes number as follows:

$$
C_{T D}=C_{\mu}^{1 / 4} \frac{1}{S_{t}\left(1+S_{t}\right)} .
$$

The non-drag forces acting on the liquid phase are the same with those on the vapor phase but in the opposite direction.

\subsubsection{Interfacial Heat and Mass Transfer}

The interfacial energy transfer terms, $Q_{i g}$ and $Q_{i l}$, in Eqs. (4) and (5) are modeled as:

$$
\begin{gathered}
\left.Q_{i g}=\frac{P_{s}}{P} H_{i g} a_{i}\left[T_{s a t}\left(P_{s}\right)-T_{g}\right)\right]+\Gamma_{g} h_{g i}, \\
Q_{i l}=H_{i l} a_{i}\left[T_{s a t}\left(P_{s}\right)-T_{l}\right]-\Gamma_{g} h_{l i},
\end{gathered}
$$

where the first terms in the right-hand side of Eqs. (27) and (28) are the bulk interface heat transfer and the second terms are the interface energy transfer due to an interface mass transfer. $P_{s}$ is the steam partial pressure. The interface enthalpies $h_{g i}$ and $h_{l i}$ are defined in such a way that the interface energy jump conditions at a liquid-vapor interface are satisfied [14]. Because the sum of $Q_{i g}$ and $Q_{i l}$ is zero, the volumetric vapor generation rate is represented as:

$$
\Gamma_{g}=-\frac{\left.\left.\frac{P_{s}}{P} H_{i g} a_{i}\left[T_{s a t}\left(P_{s}\right)-T_{g}\right)\right]+H_{i l} a_{i}\left[T_{s a t}\left(P_{s}\right)-T_{l}\right)\right]}{h_{g i}-h_{l i}} .
$$

The term $Q_{g l}$ in Eqs. (4) and (5) is the sensible heat transfer rate per unit volume at the noncondensable gas- 
liquid interface:

$$
\begin{aligned}
Q_{g l} & =\frac{P-P_{s}}{P} H_{g l} a_{i}\left(T_{g}-T_{l}\right) \\
& =\frac{P_{n}}{P} H_{g l} a_{i}\left(T_{g}-T_{l}\right),
\end{aligned}
$$

where $H_{g l}$ is the sensible heat transfer coefficient, and $P_{n}$ is the noncondensable gas partial pressure. The multiplier based on the pressures is an ad hoc function used to turn off this term when there is no noncondensable gas [14].

The interfacial heat transfer coefficients for each topology are listed in Table 2. When the liquid temperature is greater than the saturation temperature, the liquid-side heat transfer is calculated using the following approximate model:

$$
H_{i l} a_{i}=\max \left(10^{6}, H_{i l} a_{i}\right) .
$$

This model was adopted to quickly decrease the liquid temperature to saturation temperature.

\subsection{Turbulent Shear Stress}

For the turbulent shear stress, two models were implemented; one is the mixing length model and the other the standard k- $\varepsilon$ model. Considering the effect of bubbles on the turbulence structure, the bubble induced turbulence terms were included in both models, which were proposed by Michiyochi and Serizawa [32] and Sato et al.[33], respectively.

The standard wall function was used for the singlephase flow simulation. However, in a two-phase flow, it was reported that the existence of bubbles on the wall boundary layer has a significant influence on the velocity profile nearby the wall. The two-phase wall function proposed by Yun et al. [34], therefore, was implemented for the boiling flow simulation accounting for this effect.

\subsection{Heat Structure and Wall Heat Transfer}

In many transient situations of a nuclear reactor, a thermal analysis for a structure exchanging heat with fluids is as important as that for a two-phase flow. A conjugate heat transfer analysis between a fluid and a heat structure, therefore, is required for the CUPID code. It uses a three-dimensional heat conduction equation for heat structures:

$$
\begin{gathered}
\int \rho_{\text {solid }} C_{P, \text { solid }} \frac{\partial T_{\text {solid }}}{\partial t} d V=\int \nabla \cdot k_{\text {solid }} \nabla T_{\text {solid }} d V \\
+\int q_{\text {solid }}^{\prime \prime \prime} d V+\int q_{\text {fluid -solid }}^{\prime \prime} d A .
\end{gathered}
$$

\begin{tabular}{|c|c|}
\hline Local flow regime & Interfacial heat transfer coefficient \\
\hline Bubble & $H_{i k}=\frac{k_{k} N u_{b}}{D_{b}}$ where $N u_{b}=2+0.6 \operatorname{Re}_{b}^{0.5} \operatorname{Pr}_{l}^{0.3}$ \\
\hline Mist & $H_{i k}=\frac{k_{k} N u_{d}}{D_{d}}$ where $N u_{d}=2+0.6 \operatorname{Re}_{d}^{0.5} \operatorname{Pr}_{g}^{0.3}$ \\
\hline Sharp interface & $\begin{array}{c}H_{i k}=S t \rho_{k} c_{p, k}\left|\underline{u}_{\tau, g}-\underline{u}_{\tau, l}\right| \\
\text { where } S t=0.0045\left(\frac{\rho_{g}\left|\underline{u}_{g}\right| \mu_{l}}{\rho_{l}\left|\underline{u}_{l}\right| \mu_{g}}\right)^{1 / 3} \text { and } \\
\underline{u}_{\tau, g}-\underline{u}_{\tau, l}=\left(\underline{u}_{g}-\underline{u}_{l}\right)-\left[\left(\underline{u}_{g}-\underline{u}_{l}\right) \cdot \frac{\nabla \alpha_{g}}{\left|\nabla \alpha_{g}\right|}\right] \frac{\nabla \alpha_{g}}{\left|\nabla \alpha_{g}\right|} .\end{array}$ \\
\hline Transition & $\left(H_{i k} a_{i}\right)_{\text {transition }}=w \cdot\left(H_{i k} a_{i}\right)_{1}+(1-w) \cdot\left(H_{i k} a_{i}\right)_{2}$ \\
\hline
\end{tabular}

Eq. (32) was explicitly coupled with the fluid energy equations of each phase to simulate the heat transfer

Table 2. The Interfacial Heat Transfer Models in the CUPID Code 
between the structure and fluids. With the finite volume discretization, the governing equation can be solved on the three-dimensional unstructured meshes.

In order to distribute the heat from the structure to each phase, a heat partitioning model is necessary:

$$
q_{\text {fluid-solid }}=q_{\text {fluid-solid }}^{\prime \prime} A=q_{w l}+q_{w g} .
$$

If the interface topology in the wall adjacent cell is the bubbly flow, the mechanisms of a heat transfer from the wall to a two-phase flow was assumed to consist of the surface quenching $\left(q_{q}\right)$, evaporation $\left(q_{e}\right)$ and single phase convection $\left(q_{w l c}\right.$ and $\left.q_{w g}\right)$. The wall-to-liquid heat transfer $q_{w l}$ in Eq. (33) is the sum of $q_{q}$ and $q_{w l c}$. If the mist topology is chosen, it was assumed that all heat from the wall is transferred to gas through convection and the direct contact heat transfer between droplets and the wall is negligible. In the transition region, the heat from the wall is distributed to each phase by the linear interpolation between the bubbly flow and the mist flow according to the void fraction. Then, the equation of the heat conservation on a heated surface for the transition region is

$$
\begin{aligned}
q_{\text {fluid-solid }} & =\frac{\alpha_{g, c m}-\alpha_{g}}{\alpha_{g, c m}-\alpha_{g, b c}}\left(q_{q}+q_{e}+q_{w l c}\right) \\
& +\frac{\alpha_{g}-\alpha_{g, b c}}{\alpha_{g, c m}-\alpha_{g, b c}} q_{w g} .
\end{aligned}
$$

Various closure relations were implemented for each term of Eq. (34) and they are listed in Table 3.

\section{NUMERICAL SOLUTION METHODS}

In the CUPID code, the finite volume method is applied to the integration of the two-fluid governing equations on unstructured grids. All the system variables of pressure, velocities, volume fractions, and energies are defined at a cell center. The cell-faced values are interpolated using the cell-centered values. Figure 2 shows an example of an unstructured grid in two dimensions, where $f$ is the face number of cell $\mathrm{c} 0$, and $\underline{S}_{f}$ is the area vector of the face $f$ between cell $\mathrm{c} 0$ and $\mathrm{c} 1$.

\subsection{A Semi-Implicit Two-Step Numerical Solution Scheme}

The solution algorithm of the CUPID code was originally developed from the semi-implicit method for a two-phase flow [14, 37] with some modifications for an

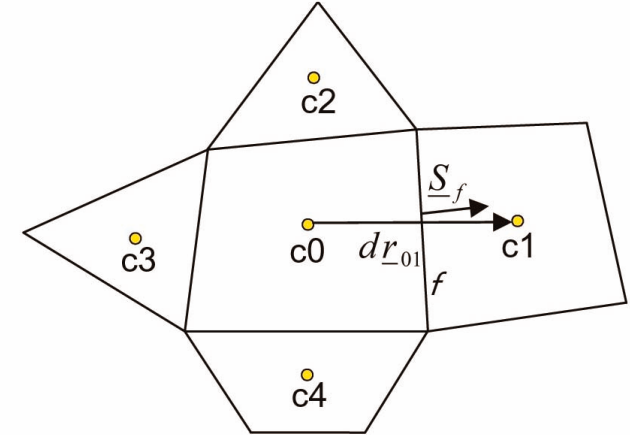

Fig. 2. Control Volumes for the Finite Volume Discretization

application to unstructured non-staggered grids. The method was further improved for enhanced robustness and accuracy [38], finally resulting in a semi-implicit two-step method.

It is noted that, in the CUPID code, a semi-conservative form of the phasic momentum equation is used instead of the conservative form in Eq. (3), which is closer to the conservative form but still maintains the numerically convenient feature of the non-conservative form (See Section 3.2.1):

$$
\begin{gathered}
\alpha_{k} \rho_{k} \frac{\partial \underline{u}_{k}}{\partial t}+\nabla \cdot\left(\alpha_{k} \rho_{k} \underline{u}_{k} \underline{u}_{k}\right)-\underline{u}_{k} \nabla \cdot\left(\alpha_{k} \rho_{k} \underline{u}_{k}\right) \\
+\underline{u}_{k} \Omega_{k}=-\alpha_{k} \nabla P+\nabla \cdot\left[\alpha_{k}\left(\tau_{k}+\tau_{k}^{T}\right)\right] \\
+\alpha_{k} \rho_{k} \underline{g}+\underline{F}_{i k}+\underline{F}_{k}^{N D} .
\end{gathered}
$$

\subsubsection{Explicit Momentum Calculation}

The momentum equations are integrated over a cell $\mathrm{i}$ in an unstructured grid. Only the interface momentum transfer terms in Eqs. (35) are treated implicitly. The discretized equations for the three fields are spatially not linked and, thus, three simultaneous linear equations are obtained with three unknown phasic velocities, $\underline{u}_{k, i}^{*}$, where the superscript $*$ indicates a temporal value. After solving the phasic momentum equations, we obtain the temporal phasic velocity represented by:

$$
\underline{u}_{k, i}^{*}=\underline{\gamma}_{k, i}^{n}+\beta_{k, i} \nabla P_{i}^{n}
$$

where $\gamma_{k, i}^{n}$ includes the explicit convection, diffusion, and body force contributions and $\beta_{k i}$ is the coefficient of the 
pressure gradient. The new velocity is implicitly linked to a new pressure from the momentum equation as given by

$$
\underline{u}_{k, i}^{n+1}=\underline{\gamma}_{k, i}^{n}+\beta_{k, i} \nabla P_{i}^{n+1} \text {. }
$$

Subtracting Eq. (36) from Eq. (37) we obtain:

$$
\underline{u}_{k, i}^{n+1}=\underline{u}_{k, i}^{*}+\beta_{k, i} \nabla P_{i}^{\prime},
$$

where $P_{i}^{\prime}\left(=P_{i}^{n+1}-P_{i}^{n}\right)$ is a pressure correction which will be determined from the mass and energy conservation equations. To get an expression for the cell face phasic volume flow, $\Psi_{k, f}^{n+1}=\underline{u}_{k, f}^{n+1} \cdot \underline{S}_{f}$, we apply a divergence operator of $\nabla$. to Eq. (38) and integrate it over the computing cell $i$. Then, the volume flow of $\Psi_{k f}^{n+1}$ at the cell face $f$ is obtained as:

$$
\sum_{f} \Psi_{k, f}^{n+1}=\sum_{f}\left[\Psi_{k, f}^{*}+\beta_{k, f} \frac{A_{f}}{\left|\underline{d r_{f}}\right|}\left(P_{j}^{\prime}-P_{i}^{\prime}\right)\right] .
$$

\begin{tabular}{|c|c|c|}
\hline Variables & Models & References \\
\hline Surface quenching & $q_{q}=\left(\frac{2}{\sqrt{\pi}} \sqrt{t_{w} k_{l} \rho_{l} C_{p l}} f\right) A_{2 f}\left(T_{w}-T_{l}\right)$ & [35] \\
\hline Wall boiling & $q_{e}=N^{\prime \prime} f\left(\frac{\pi}{6} D_{b, \text { depart }}^{3}\right) \rho_{g} h_{f g}$ & [35] \\
\hline Single phase convection & $q_{c}=h_{c} A_{c}\left(T_{w}-T_{c}\right)$ & [35] \\
\hline $\begin{array}{l}\text { Active nucleate } \\
\text { site density }\end{array}$ & $N^{\prime \prime}=\left[185\left(T_{w}-T_{\text {sat }}\right)\right]^{1.805}$ & [35] \\
\hline $\begin{array}{l}\text { Bubble departure } \\
\text { frequency }\end{array}$ & $f=\sqrt{\frac{4 g\left(\rho_{l}-\rho_{g}\right)}{3 D_{b, d e p a r t} \rho_{l}}}$ & [35] \\
\hline $\begin{array}{l}\text { Bubble departure } \\
\text { diameter }\end{array}$ & $D_{b, \text { depart }}=1.5 \cdot 10^{-4} \sqrt{\frac{\sigma}{g \Delta \rho}}\left[\frac{\rho_{l} c_{p l}\left(T_{\text {wall }}-T_{\text {sat }}\right)}{\rho_{g} h_{\mathrm{lg}}}\right]^{5}$ & $\begin{array}{c}\text { Cole and } \\
\text { Rosenhow [36] }\end{array}$ \\
\hline Bubble waiting time & $t_{w}=0.8 / f$ & [35] \\
\hline Heat transfer coefficient & $h_{c}=S t \cdot \rho_{c} \cdot C_{p c} \cdot u_{\tau}$ & - \\
\hline $\begin{array}{l}\text { Single phase heat } \\
\text { transfer area }\end{array}$ & $A_{1 f}=1-A_{2, f}$ & [35] \\
\hline $\begin{array}{c}\text { Two-phase heat transfer } \\
\text { area }\end{array}$ & $A_{2 f}=N^{\prime \prime} \frac{\pi D_{b, \text { depart }}^{2}}{4} K$ & [35] \\
\hline Bubble influence factor & $K=4$ & [35] \\
\hline
\end{tabular}

Table 3. Closure Relations for the Wall Heat Transfer 


\subsubsection{Predictor Step}

Combining the mass conservation equations, we obtain:

$$
\begin{gathered}
\frac{1}{V_{i}} \sum_{f} \frac{1}{\rho_{k}} \sum_{k}(\alpha \rho)_{k, f} \Psi_{k, f}^{n+1}=\Gamma_{g}^{n+1} \\
\left(\frac{1}{\rho_{g}}-\frac{1}{\rho_{l}}\right)-\frac{\alpha_{g}}{\rho_{g}} \frac{\rho_{g}^{\prime}}{\Delta t}-\frac{\left(1-\alpha_{g}\right)}{\rho_{l}} \frac{\rho_{l}^{\prime}}{\Delta t},
\end{gathered}
$$

where variables with no superscript are the old values. The phase change rate, $\Gamma_{g}^{n+1}$, is linearized with respect to the energy, noncondensable gas quality and pressure variations. Eq. (39) is inserted into Eq. (40) to eliminate $\Psi_{k, f}^{n+1}$. Then the pressure equation becomes

$$
D_{i} P_{i}^{\prime}+\sum_{f} D_{f} P_{j}^{\prime}=D_{i}^{S}
$$

where $D_{i}, D_{f}$, and $D_{i}^{S}$ are coefficients of the pressure matrix [38]. The phasic density differences between neighboring cells are very small so that the coefficient matrix of Eq. (41) is actually symmetric.

\subsubsection{Update the Interfacial Heat Transfer Coefficients}

The volumetric phase fraction can be found from the combined continuity equation using the phasic volume flow and pressure calculated from Eqs. (39) and (41):

$$
\sum_{k=v, l, d}\left[\frac{V_{i}}{\Delta t}\left(\alpha_{k}^{m} \rho_{k}^{m}-\alpha_{k}^{n} \rho_{k}^{n}\right)+\sum_{f}\left(\alpha_{k}^{n} \rho_{k}^{n} \Psi_{k}^{m}\right)_{f}\right]=0
$$

where $\rho_{k}^{n} \approx \rho_{k}^{n}+\frac{\partial \rho_{k}}{\partial P} \delta P+\frac{\partial \rho_{k}}{\partial e_{k}} \delta e_{k}$. Old time values are used for $\delta e_{k}$ since the energy equation is not solved yet. This is why the calculated phase fractions are the predictor of new time value. The superscript $m$ indicates the predictor step. The interfacial heat transfer coefficient is updated using the predicted phase fractions:

$$
H_{i k}=H_{i k}\left(\alpha_{k}^{m}\right) \text {. }
$$

\subsubsection{Corrector Step}

The six scalar equations are integrated over a computing cell. In this step, the convection terms are treated using implicit velocities while the convected quantities such as the void fraction and density are treated explicitly. For the non-linear terms, first order Taylor series expansions at the old time step are used to linearly obtain variables at new time step. These linearized equations are rearranged with respect to six independent scalar variables $\left(e_{g}^{\prime}, e^{\prime}{ }_{l}, \alpha_{g}^{\prime}, \alpha_{d}^{\prime}, X_{n}^{\prime}, P^{\prime}\right)$ as:

$$
\underline{\underline{A}}_{i} \underline{x}_{i}=\underline{S}_{i}+\sum_{f} \sum_{k} \underline{\underline{c}}_{k, f} \Psi_{k, f}^{n+1}
$$

where $A_{i}$ is a $6 \times 6$ coefficient matrix obtained from the above-mentioned six scalar equations, and $\underline{x}_{i}$ is the solution vector, and $\underline{s}_{i}$ and $\underline{c}_{k, f}$ are coefficient vectors.

The solution vector is obtained by multiplying $A_{i}^{-1}$ to Eq. (44) as follows:

$$
\underline{x}_{i}=\underline{\underline{A}}_{i}^{-1} \underline{S}_{i}+\sum_{f} \underline{A}_{i}^{-1} \sum_{k} \underline{c}_{k, f} \Psi_{k, f}^{n+1}
$$

All the six scalar equations are used to obtain the pressure correction equation. The pressure equation is obtained by substituting Eq. (39) in the sixth row of Eq. (45) and eliminating $\Psi_{k, f}^{n+1}$. An iterative method like the conjugate gradient method could be used to get a solution from the linear equation. After the pressure equation is solved, the new time phasic velocity $\underline{\mathrm{u}}_{k, i}^{n+1}$ and volume flow $\Psi_{k, f}^{n+1}$ are determined from Eqs. (37) and (39). Finally, the scalar variables are updated from Eq. (45) and the remaining state variables such as densities, temperatures, etc. are calculated using the steam table functions.

In a two-phase fluid flow, thermal non-equilibrium transient calculations are hard to converge and the interfacial heat transfer coefficient has to be used very carefully. Since the interfacial heat transfer coefficient is nearly implicit in the present two-step method, it greatly enhances numerical stability, especially, when two phases are in thermal non-equilibrium. The pressure calculation for the corrector-step is not necessary when the two-phase flow is in steady-state or thermal-equilibrium conditions.

\subsection{Some Numerical Improvements}

To enhance the accuracy and robustness of the CUPID numerical method, some improvements were made. Detailed descriptions were given in References [39 41].

\subsubsection{The Semi-Conservative Form of Momentum Equations}

The CUPID code used a non-conservative form of 
the two-phase momentum equation as follows:

$$
\begin{gathered}
\alpha_{k} \rho_{k} \frac{\partial \underline{u}_{k}}{\partial t}+\alpha_{k} \rho_{k} \underline{u}_{k} \cdot \nabla \underline{u}_{k}+\underline{u}_{k} \Omega_{k}=-\alpha_{k} \nabla P \\
+\nabla \cdot\left[\alpha_{k}\left(\tau_{k}+\tau_{k}^{T}\right)\right]+\alpha_{k} \rho_{k} \underline{g}+\underline{F}_{i k}+\underline{F}_{k}^{N D} .
\end{gathered}
$$

This non-conservative form is good for numerical convenience, especially for the time advancement of phasic velocity only. But, it might evoke greater numerical errors in some multi-dimensional flow simulations. This can be substantially improved by using a semi-conservative form of equations, which is represented in Eq. (35). The semi-conservative form in Eq. (35) is still non-conservative in time but conservative in space. Eq. (35) reduces to Eq. (3) in a steady state, in other words, the semi-conservative form becomes a conservative form in the case of a steady state. Its numerical advantages were presented by Park et al. [39].

\subsubsection{A Second-Order Upwind Scheme for Convective Terms and Diffusion Terms}

In the earlier versions of the CUPID code, the convective terms were calculated by the first-order upwind scheme. To enhance the numerical accuracy, a secondorder upwind scheme was developed [40]. The convective terms in the governing equations are discretized as follows:

$$
\int \nabla \cdot\left(\theta \underline{u}_{k}\right) d V \approx \sum_{f}(\theta)_{f}\left(\Psi_{k}\right)_{f}
$$

where $(\theta)$ represents convective quantities such as $\left(\alpha_{k} \rho_{k}\right)_{f}$, $\left(\alpha_{k} \rho_{k} \underline{u}_{k}\right)$, and $\left(\alpha_{k} \rho_{k} e_{k}\right)_{f}$, for the mass, momentum, and energy equations, respectively. These convective quantities are assumed to be distributed in a piecewise continuous manner, which represents the data on either side of a cell face may be discontinuous. The unique convective quantity at a cell face is then obtained by using an upwind method from two distinct convective quantities, $\theta$ and $\theta^{*}$, depending on the sign of normal velocity at a cell face.

For the first-order upwind scheme, the data are assumed to be constantly distributed in a cell, consequently two convective quantities on a face can be directly obtained from the values at the cell centers. In the second-order upwind scheme, the assumption of piecewise constant distributions is replaced by a linear distribution. Therefore, the convective quantities on the face are given by:

$$
(\theta)_{f}=\left\{\begin{array}{lll}
\theta^{-}=\theta_{i}+(\nabla \theta)_{i} \cdot \underline{d x}_{f i} & \text { if } \quad(\Psi)_{f} \geq 0 \\
\theta^{+}=\theta_{j}+(\nabla \theta)_{j} \cdot \underline{d x}_{f j} & \text { if } \quad(\Psi)_{f}<0
\end{array},\right.
$$

where $\underline{d x}_{f k}$ represents the vector extending from the cell center to the center of the cell face. Equation (47) requires the evaluation of the gradient $\nabla \theta$ at the cell center. This gradient can be reconstructed by using a Green-Gauss theorem or by taking a least-square approximation to the neighboring cells. Among those, the Frink's method, originally developed on tetrahedral unstructured meshes [42], was modified to evaluate the gradients on arbitrary polyhedral cells. In this method, the data are interpolated to the nodes first and the gradient then is evaluated by applying the Green-Gauss theorem along the path of a cell. Since the Frink's method has the two interpolation procedures, from cell centers to a node and Green-Gauss integration, the method utilizes the increased support of the stencil and consequently smoothens the computed gradient for a highly distorted cell, thus enhances the stability of the overall scheme.

The first-order upwind scheme can obtain stable solutions without non-physical oscillations in the presence of discontinuities, due to the monotonicity properties on arbitrary polygonal control volumes [43]. However, it is well known that high-order numerical schemes produce spurious oscillations in the vicinity of discontinuities, which can lead to numerical instabilities and unbounded solutions. To stabilize numerical solutions of the secondorder scheme for two-phase flows where discontinuities might be present in convective quantities, a slope limiter approach which was originally developed for capturing shock waves for compressible flows was adopted. In the context of unstructured finite volume frameworks, two multi-dimensional slope limiters of Barth and Jesperson [43] and Venkatakrishnan [44] have been widely used. To enhance the versatility, we implemented the two limiters.

Initially, the diffusion terms were also approximated with first-order accuracy. Later, the accuracy was enhanced to a second-order. The diffusion terms in the governing equations are discretized as follows:

$$
\int \nabla \cdot\left(\theta \nabla \underline{u}_{k}\right) d V=\sum_{f} \theta_{f}(\nabla \phi \cdot \underline{n})_{f},
$$

where $\theta_{f}$ represents diffusive face normal gradient such as $\left(\alpha_{k} \mu_{k}\right)_{f}$ and $\left(\alpha_{k} k_{k}\right)_{f}$ for the momentum and energy equations, respectively. $(\nabla \phi \cdot \underline{\mathrm{n}})_{f}$ represents the face normal gradient and it is obtained by using the methods of Weiss [45] and Mathur and Murthy [46]. In the Weiss method, the face normal gradient is given as:

$$
(\nabla \phi \cdot \underline{n})_{f}=\overline{\nabla \phi} \cdot \underline{n}-\left[\bar{\nabla} \phi \cdot \underline{r}_{, j}-\frac{\Delta \phi_{i, j}}{\left|r_{i, j}\right|}\right] \underline{r}_{, j} \cdot \underline{n} .
$$


In the Mathur and Murthy method, it is derived as:

$$
(\nabla \phi \cdot \underline{n})_{f}=\bar{\nabla} \phi \cdot \underline{n}-\left[\overline{\nabla \phi} \cdot \underline{r}_{i, j}-\frac{\Delta \phi_{i, j}}{\left|\underline{r}_{i, j}\right|}\right] \frac{1}{\underline{r}_{, j} \cdot \underline{n}},
$$

where the mean gradient $\bar{\nabla}$ was calculated as the average of the gradient at the own cell $\mathrm{i}$ and the neighboring cell $\mathrm{j}$, the cell gradient was calculated by the Frink's reconstruction method, and $\underline{r}_{i, j}$ is a unit direction vector.

\subsubsection{An Improved Scheme for the Pressure Gradient Calculation}

In the CUPID code [10], the pressure gradient at a cell center was evaluated by using the Green-Gauss reconstruction method:

$$
\nabla P_{0}=\frac{1}{V_{0}} \sum_{f} P_{f} \underline{n}_{f} \underline{S}_{f}
$$

where the subscript $f$ indicates the faces of the cell 0 . In the case of a two-dimensional mesh, the pressure at the face $f$ was determined by using an inverse-distance weighting. This method, i.e., the Green-Gauss reconstruction with an inverse-distance weighting, has second-order accuracy on structured meshes, but may have some error for skewed unstructured meshes. To overcome this problem, the Frink's reconstruction method, which was also adopted for obtaining the second-order accuracy of convective terms on unstructured meshes, is used in the new version.

The reconstruction of the pressure gradient at a boundary cell is somewhat different from that at inner cells because of the interpolation error of the pressure at the boundary face. For example, when the Green-Gauss reconstruction method is used, the pressure gradient at a boundary cell can be underestimated if the pressure at the boundary face is assumed to be equal to that at the boundary cell center. To mitigate this error, the pressure gradient at the boundary cell was evaluated by the least-square reconstruction method in the previous CUPID code. Even though this method greatly enhances the accuracy of the pressure gradient at the boundary cells, the results of some numerical tests showed non-negligible error at the boundary cells of skewed unstructured meshes.

To enhance the accuracy of pressure gradient at boundary cells on unstructured meshes, a new iterative method based on the Frink's method was developed [41]. In this method, initial pressure value at a boundary node is evaluated by using pseudo-Laplacian weighting method and used to reconstruct the pressure gradients at cells connected to the boundary node, as in the original Frink's method. Then new pressure value at the boundary node is extrapolated with the reconstructed pressure gradients at boundary cells. This boundary-node pressure is recursively utilized for the evaluation of pressure gradients at boundary cells. This iteration continues until all extrapolated pressures at the boundary nodes converge. Finally, the pressure gradients at the boundary cells are evaluated by using the Frink's method with the converged pressures at the boundary nodes.

The new iterative method was compared with various gradient calculation methods in the literatures by using a simple test function, a gravity-driven cavity, and a wall boiling two-phase flow problems [41]. The results of the calculations clearly showed that the new iterative pressure gradient evaluation method leads to the most accurate results for both the structured and unstructured grids under single- and two-phase flow conditions.

\section{CODE COUPLINGS}

The CUPID code was designed as a standalone code, but coupled with a system code and a three-dimensional reactor kinetics code. This allows for an advanced simulation of nuclear thermal hydraulics as well as a wider range of the code applications.

\subsection{Coupling with the System Thermal-Hydraulics Code, MARS}

Since the CUPID code is a macroscale code, the coupling with a system code is conceptually straightforward. By using the coupled code, we can simulate a specific part of a thermal-hydraulic system with CUPID and the others with the system code, which can provide the system code with a local zoom function. The system code, MARS [15], was chosen for this coupling because its hydrodynamic model adopts a two-fluid model for two-phase flow and its numerical scheme is similar to the corrector step of the CUPID code. Referring to the previous experiences [15, 47], the MARS-CUPID coupling was progressed.

Let us consider a flow system that is divided into a three-dimensional region (modeled by CUPID) and several one-dimensional regions (modeled by MARS) with $N C$ interface junctions (or connections). For convenience, let us define $C i$ and $M i$ as the index numbers of $i$-th interfacing cells in the CUPID and MARS regions, respectively. Concept and assumptions for the code coupling are

(a) The momentum equations at the interface junction are solved by the MARS code.

(b) It is assumed that continuous liquid and entrained droplets at the interface are in mechanical equilibrium, i.e., $V_{d}=V_{l}$. 
(c) In the MARS code, cell $\mathrm{Ci}$ is treated as a "CUPID boundary volume (cupvol)", whose scalar variables are updated every time-step by CUPID. When the flow enters cell $M i$ from cell $C i$, continuous liquid and droplets are agglomerated into the liquid phase at the interface.

(d) In the CUPID code, cell $M i$ is regarded as a sink that is implicitly coupled. When liquid enters cell $\mathrm{Ci}$ from cell $\mathrm{Mi}$, the liquid phase is divided into continuous liquid and droplets at the interface according to a partition rule [15]. The rule directly uses some correlations for entrainment fraction calculation in annular-mist flow regime.

(e) The pressure correction matrices, which are set up in each code, are coupled via the momentum modeling at the interfaces and solved simultaneously.

The momentum balance at the interface junction from cell $C i$ to $M i$ is modeled by the MARS code, where the old time-step variables of cell $C i$ are transferred from CUPID. Then, the phasic velocity at the i-th interface junction $V_{k, i}^{n+1}$ is given by

$$
V_{k, i}^{n+1}=\alpha_{k, i}+\beta_{k, i}\left(\delta P_{C i}-\delta P_{M i}\right)
$$

Effects of the connections should be taken into account for the conservation of momentum in the CUPID region.

Because of the MARS-CUPID connections, the pressure correction equation of MARS involves additional unknown terms that include the unknown velocities at the interfaces:

$$
\underline{\underline{A}}_{M} \delta \underline{P}_{M}=\underline{b}_{M}+\sum_{i=1}^{N C}\left(\underline{\gamma}_{f, i} V_{f, i}^{n+1}+\underline{\gamma}_{g, i} V_{g, i}^{n+1}\right)
$$

where $\underline{\gamma}_{f}$ and $\underline{\gamma}_{g}$ are coefficient vectors. Likewise, the pressure correction equation of CUPID is also changed due to the connection:

$$
\underline{\underline{A}}_{C} \delta \underline{P}_{C}=\underline{b}_{C}+\sum_{i=1}^{N C}\left(\underline{\kappa}_{f, i} V_{f, i}^{n+1}+\underline{\kappa}_{g, i} V_{g, i}^{n+1}\right)
$$

where $\underline{\kappa}_{f}$ and $\underline{\kappa}_{g}$ are coefficient vectors. Inserting Eq. (52) into Eqs. (53) and (54), a coupled pressure correction equation for the whole system can be established. The coupled equation is solved by using a domain decomposition method. After solving it, the remaining numerical sequences are completed in each code. Figure 3 shows a simple example of MARS-CUPID coupling and the coupled pressure correction matrix. Currently the basic coupling has been completed but the verificaion is still being carried out.

\subsection{Coupling with the Three-Dimensional Reactor Kinetics Code, MASTER}

In order to provide accurate predictions in the analysis of system transients involving strong interactions between neutron and thermal-hydraulic phenomena, the coupled codes $[48,49]$ have been developed. This is especially important when asymmetric behavior appears in the reactor core. In this regard, the CUPID code was coupled with the three-dimensional reactor kinetics code, MASTER [49], for the possible applications to a nuclear reactor core.

The coupling of CUPID and MASTER was achieved under the Windows operating system so that the use of the dynamic link library (DLL) feature could be used and the code can be executable on personal computers. The use of DLL allowed maintaining the integrity of each code independently as well as keeping simpler coupled code structures. Only minor code changes were needed for data communication and for incorporating feedback data. In the coupled code, the transient core thermalhydraulic conditions, such as coolant density and fuel temperature, are determined at each time step by CUPID and transferred to MASTER so that they can be used to update the group constants. The power distribution newly obtained by MASTER is then sent back to CUPID.

To verify the performance of the coupled code, the reactor core of a pressurized water reactor $\left(2815 \mathrm{MW}_{\text {th }}\right)$ at a steady-state condition was simulated. Then, the transient of a single control element assembly (CEA)

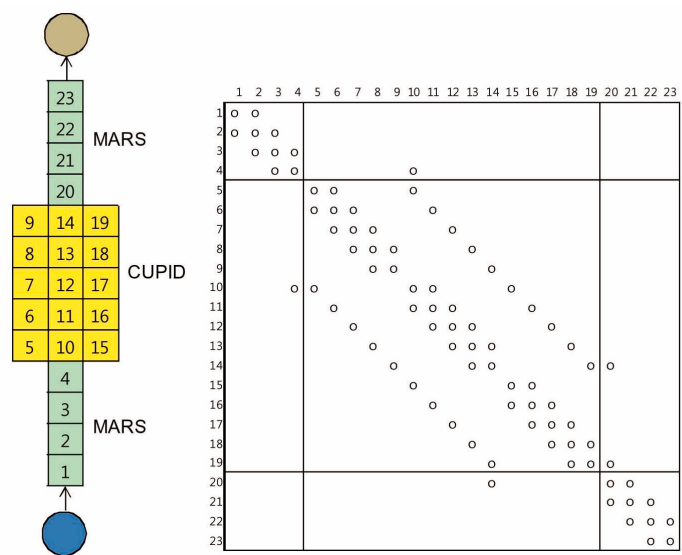

Fig. 3. An Example of the MARS-CUPID Coupling and the Coupled Pressure Correction Matrix 


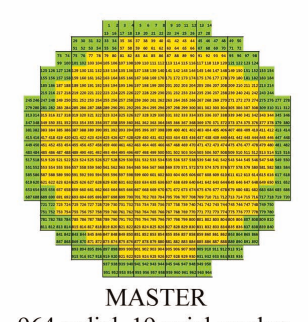

964 radial, 19 axial meshes

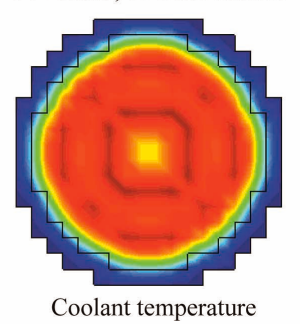

before the CEA drop $(\mathrm{K})$

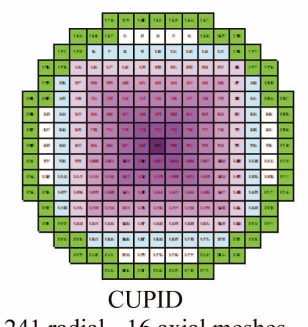

241 radial -16 axial meshes

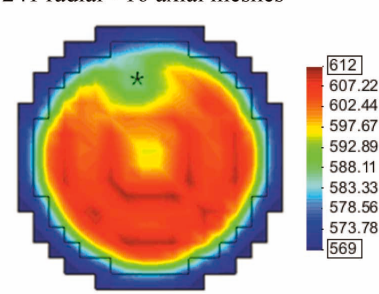

Coolant temperature after the CEA drop (K)

Fig. 4. An Example of the CUPID-MASTER Coupled

Calculation: The Computational Meshes and Coolant

Temperature at the Core Exit (*The location of the Dropped CEA)

drop was calculated $[50,51]$. The transient leads to an asymmetric core behavior, where the role of a coupled three-dimensional calculation is crucial. Figure 4 show the computational meshes and the coolant temperature at the core exit before and after the CEA drop; the results show that the coupled code works qualitatively well. Quantitative assessment is to be carried out later.

\section{THE STRATEGY AND STATUS OF THE CODE VERIFICATION AND VALIDATION}

Two key steps for code development are verification and validation. The verification is the assessment of the software correctness and numerical accuracy of the solution to a given computational model. Meanwhile, the validation is the assessment of the physical accuracy of a computational model based on comparisons between computational simulations and experimental data. This section introduces the status and strategy of the CUPID code verification and validation.

\subsection{Verification}

The CUPID code was systematically verified using thirteen conceptual problems, which are listed in Table 4 [11]. Each of the problems was designed so that a specific term in the governing equations or a specific algorithm can be verified. The problems are divided into four groups and the following conclusions can be drawn from the verification process:

a) Steady-state flow problems were used to confirm the correctness of the scalar equation solutions. The mass and energy flow rates were compared at the inlet and the exit of the computational domain, and the relative errors of the two-phase flows numbered less than $10^{-5}$.

b) The phase change problems were used to confirm the integrity of the numerical algorithm when a phase appears or disappears. Phase changes by boiling, flashing, and condensation were tested, leading to physically reasonable results. These problems verified the interfacial heat and mass transfer terms. The conservation equations of non-existing phases were proven to work as designed.

c) The phase separation problems were related to the momentum equations, in particular, to the interfacial drag force and gravity. In the manometric flow oscillations and the dam break problems, the phases were already separated and the hydrostatic head drives the flow transient, finally reaching an equilibrium state. Most of the terms in the momentum equations were involved in the calculations. The dam break problem was used to verify the interface surface topology concept [52]. Figure 5 shows the results of the dam break calculation. The comparison between the void fraction and the location of the sharp interface topology ensures that the topology identifying algorithm was properly implemented.

d) A three-dimensional steady-state air-water two-phase flow was simulated to confirm the continuity equations of liquid and noncondensable gas. To verify the interfacial mass transfer model under the presence of noncondensable gas, dry air $\left(X_{n}=1\right)$ was injected into the inlet of the three-dimensional duct and, then, the noncondensable gas quality $X_{n}$ along the duct was observed. $X_{n}$ should be decreased along the flow path due to the evaporation of the water vapor and its theoretical minimum is determined by the local saturation pressure of the water. The results reveal that the CUPID code works well with noncondensable gas [11].

In summary, the results of the verification show that the CUPID governing equations for two-phase flows were solved correctly on both structured and unstructured meshes.

\subsection{Validation Status and Strategy}

The validation process is a work in progress for a limited range of two-phase flow conditions [11]. Basically, the CUPID code was designed for the transient analysis of a two-phase flow at component scale and has both features of porous and open media approach. However, as mentioned previously, physical models for the two approaches are not applicable to full range of two-phase flows. Currently, the CUPID code aims at the 
Table 4. The Conceptual Problems for the Verification of the CUPID Code

\begin{tabular}{c|c}
\hline \multicolumn{1}{c|}{ Problems $^{\mathrm{a}}$} & Objectives \\
\hline $\begin{array}{c}\text { One-dimensional, steady-state liquid flow with a heat source } \\
\text { One-dimensional, steady-state steam flow } \\
\text { One-dimensional, steady-state two-phase flow } \\
\text { Two-dimensional, steady-state liquid flow }\end{array}$ & Mass and energy conservation \\
One-dimensional, boiling flow & Pho-dimensional, flashing flow change (Flow transitions) \\
Three-dimensional, boiling flow & Phase separation \\
Cavitations with a sudden contraction and a condensation & Mass conservation \\
One-dimensional, air-water phase separation by gravity & Treatment of noncondensable gases \\
Two-dimensional, air-water phase separation by gravity & Two-dimensional, air-water manometric flow oscillations \\
Three-dimensional, steady-state air-water two-phase flow &
\end{tabular}

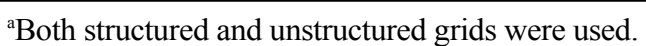

${ }^{\mathrm{b}}$ The inter-phase surface topology concept was examined.
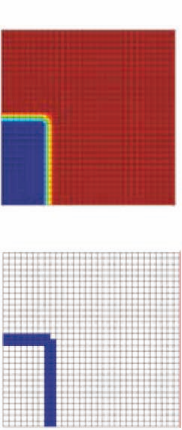

$\mathrm{t}=0.0 \mathrm{~s}$
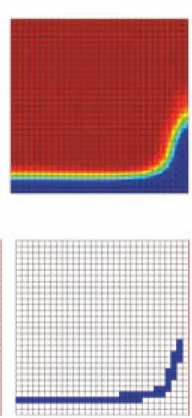

$0.3 \mathrm{~s}$

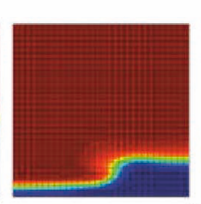

Void fraction

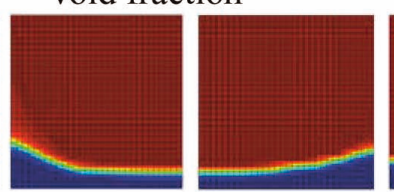

Sharp interface cells

Fig. 5. The Results of the Dam Break Calculation: Void Fraction and Sharp Interface Cells

analyses of two-phase flow mixing in a pool, downcomer boiling and emergency core coolant (ECC) bypass in a DVI system, pressurized thermal shock, etc. In recognition of this, the CUPID development and assessment strategy was established.
To date, the CUPID code has been assessed in relation to the following experiments: air-water bubbly two-phase flows in a vertical pipe (the VAWL experiment), subcooled boiling in a vertical annulus, airwater bubbly flow in a vertical slab, downcomer boiling 


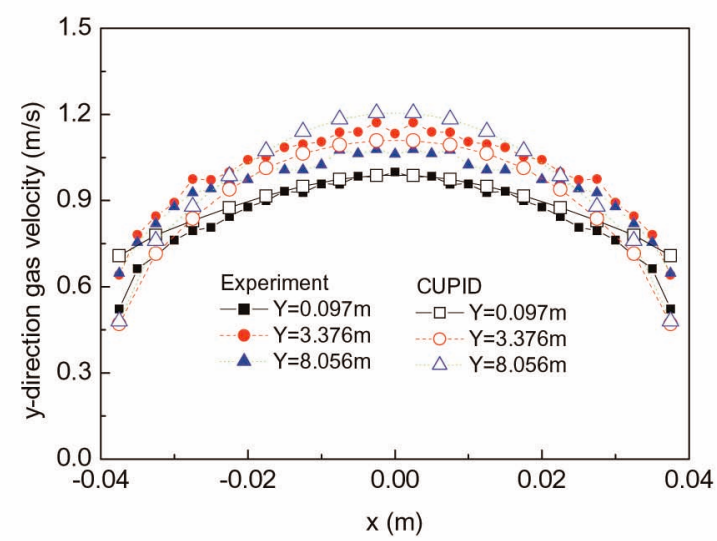

(a) Gas velocity

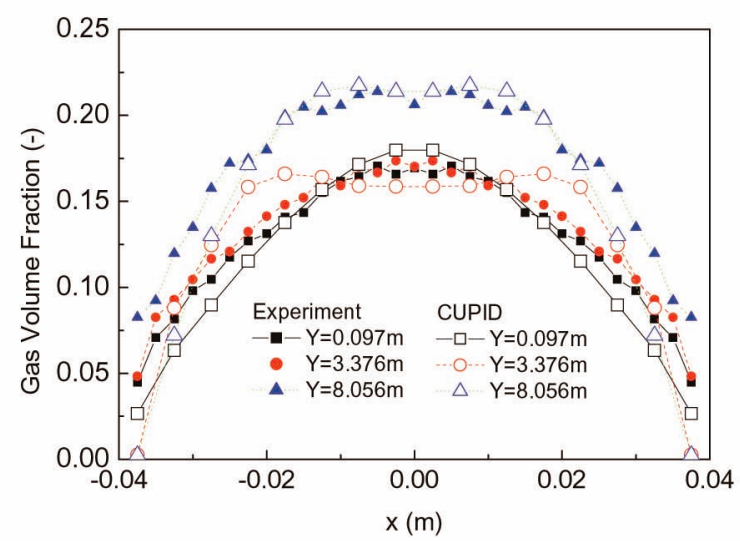

(b) Gas volume fraction

Fig. 6. Comparison of the Calculated and the Measured Radial Gas Velocity and Void Distributions of the VAWL Experiment

experiments (the DOBO experiment), and the dam break experiment [53]. In general, the assessment has shown that the CUPID code can appropriately predict these experiments, especially, under low void fraction conditions. The results of two of the cases are presented below.

The VAWL test was carried to measure the gas volume fraction and interfacial area concentration distributions of the air-water two-phase flow in a vertical pipe $[54,55]$. The test section is $0.08 \mathrm{~m}$ in diameter and $10 \mathrm{~m}$ high. Measurement stations were installed at three axial positions. For the CUPID calculation, a twodimensional grid with 16 radial, 200 axial computational meshes was used. Figure 6 shows the results of the calculation. Although the gas volume fraction profile at the center is more flat than the measurement, the nondrag forces in the CUPID code seem to work reasonably well.

The DOBO test facility [56] has a rectangular duct and its dimensions are $6.4 \mathrm{~m}$ high, $0.25 \mathrm{~m}$ wide and 0.30 $\mathrm{m}$ deep. One among four side walls of the test section incorporates 207 cartridge heaters inside it to simulate the stored energy release from the reactor vessel wall. A test was chosen for the CUPID validation. The liquid flow rate and temperature at the inlet (bottom) were 3.2 $\mathrm{kg} / \mathrm{s}(0.0183 \mathrm{~m} / \mathrm{s})$ and $111.9^{\circ} \mathrm{C}$, respectively. The heat flux from the heated wall was $72 \mathrm{~kW} / \mathrm{m}^{2}$, and the pressure at the outlet (top) was maintained at $0.16 \mathrm{MPa}$ using a control valve. The major measuring parameters were local void fraction, local gas and liquid velocities, local fluid temperature, differential pressures along the elevation, pressures, liquid flow rate, etc. In the CUPID calculation, it was assumed that the flow behavior along the depth direction is relatively negligible compared to the axial and width directions. The test was subsequently analyzed in two-dimensional approach with an 18 radial, 122 axial computational grid. Figure 7 compares the calculated and the measured radial void distributions of the DOBO experiment. A three-dimensional calculation yielded almost the same results. The results were sentitive to the bubble size model and the experiments with higher void fractions showed greater errors. The modified bubble size model [56] worked well but was not generally used to some experiments with different flow conditions. A major improvement of the interfacial transfer terms is needed for slug and churn flow regimes.

During the validation activities, shortcomings of the CUPID physical model will be specifically identified. These will be fed back to the model improvement strategy that should be devised to exclude as many empiricisms as possible and to thereby enhance CUPID's predictive capabilities.

\section{CONCLUSION}

CUPID, a three-dimensional thermal-hydraulic code was developed for the analysis of transient two-phase flows at the component scale. The CUPID code adopts a two-fluid three-field model for two-phase flows. A semiimplicit two-step numerical method was developed to obtain numerical solutions on unstructured grids. The numerical method has been further improved for enhanced accuracy and robustness.

The verification of CUPID is almost complete. A set of conceptual problems and some experiments were simulated. It was shown that the numerical scheme was accurate and robust for the predictions of single-phase 


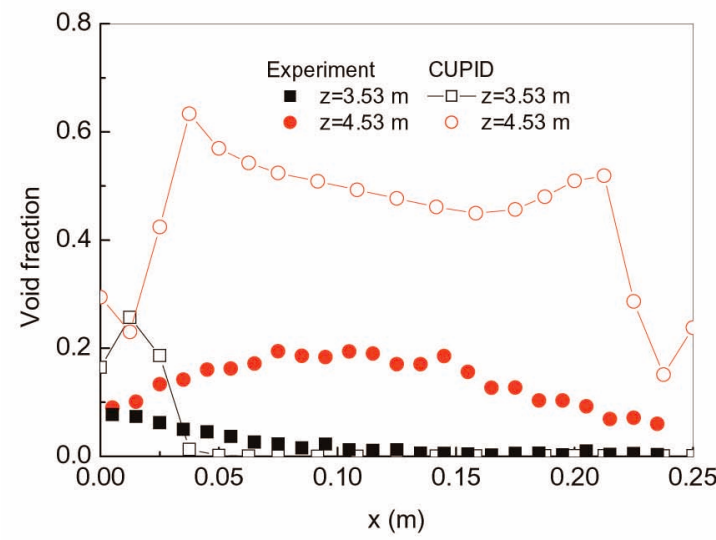

(a) The default bubble size model

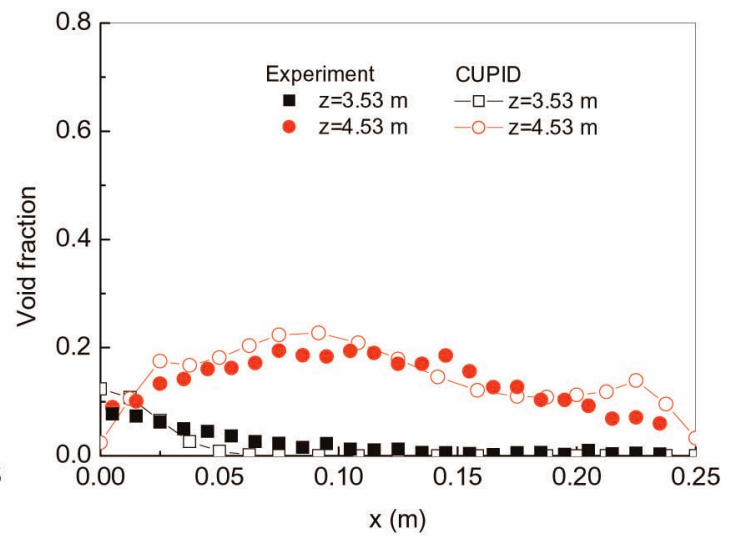

(b) The modified bubble size model

Fig. 7. Comparison of the Calculated and the Measured Radial Void Distributions of the DOBO Experiment

and two-phase flows, phase changes and flow transitions due to boiling and flashing, phase separations, and airwater two-phase flows. The conservations of the mass and energy were also confirmed.

The validation is still in progress. Currently, the physical models of the CUPID code are not applicable to wide range of two-phase flows and, thus, the assessment strategy has some limitations. To date, the CUPID code has been assessed against some experiments, of which flow conditions mainly remain at bubbly flow regime. Our results show that the CUPID code can appropriately predict these experiments, especially, under low void fraction conditions. During the future validation activities, shortcomings of the CUPID physical model are expected to be identified in detail. These will be fed back to the model improvement. Ultimately, the aim of all these activities is to develop the CUPID code as a link between a system-scale code and a finer-scale code as well as a standalone component-scale code.

\section{ACKNOWLEDGEMENT}

This work was supported by Nuclear Research \& Development Program of the NRF (National Research Foundation) grant funded by the MEST (Ministry of Education, Science and Technology) of the Korean government (Grant code: M20702040002-08M020400210).

\section{Nomenclature}

$\begin{array}{ll}a_{i} & \text { Interfacial area concentration } \\ A & \text { Surface area } \\ c_{p} & \text { Specific heat } \\ d \underline{r} & \text { Distance vector }\end{array}$

\begin{tabular}{|c|c|}
\hline$D$ & Diameter \\
\hline$e_{k}$ & Internal energy of $\mathrm{k}$-phase \\
\hline$E_{K}^{D}$ & Energy dissipation \\
\hline$F_{i k}$ & Interfacial momentum transfer \\
\hline$g$ & Gravitational acceleration \\
\hline$h$ & Enthalpy \\
\hline$H_{i k}$ & Interfacial heat transfer coefficient \\
\hline$j$ & Superficial velocity \\
\hline$k$ & Turbulence kinetic energy \\
\hline$k_{k}$ & Thermal conductivity of $\mathrm{k}$-phase \\
\hline$\underline{n}_{f}$ & Outward face normal vector \\
\hline $\bar{N}^{\prime \prime}$ & Nucleation site number density \\
\hline$N u$ & Nusselt number \\
\hline$P$ & Pressure \\
\hline $\operatorname{Pr}$ & Prandtl number \\
\hline q"' & Volumetric heat source \\
\hline$q^{\prime \prime}$ & Heat flux \\
\hline$Q_{i k}$ & Interfacial heat transfer to k-phase \\
\hline$Q_{g l}$ & Sensible heat transfer rate \\
\hline $\mathrm{Re}$ & Reynolds number \\
\hline$S_{D E}$ & Droplet de-entrainment rate per volume \\
\hline$S_{E}$ & Droplet entrainment rate per volume \\
\hline$\underline{S}_{f}$ & Surface vector \\
\hline $\bar{S}_{t}$ & Stokes number \\
\hline$S t$ & Stanton number \\
\hline$t$ & Time \\
\hline$T$ & Temperature \\
\hline$\underline{u}_{k}$ & Phasic velocity, $\underline{u}_{k}=u_{k} \underline{i}+v_{k} \underline{j}+w_{k} \underline{k}$ \\
\hline$\underline{u}_{k i}$ & Interface velocity of k-phase \\
\hline$u_{\tau}$ & Friction velocity \\
\hline$V$ & Volume or velocity \\
\hline$w$ & Weighting factor \\
\hline$\underline{x}$ & Position vector \\
\hline $\bar{X}_{n}$ & Noncondensable gas quality \\
\hline
\end{tabular}




\section{Greek Symbols}

$\alpha_{k} \quad$ Volume fraction of k-phase, where $a_{g}+a_{l}+a_{d}=1$

$\Delta t \quad$ Time step size, $t^{n+1}-t^{n}$

$\delta \varphi \quad \varphi^{n+1}-\varphi^{n}$ where $\varphi=X_{n}, e_{g}, e_{l}, \alpha_{g}, \alpha_{d}$, or $P$

$\gamma \quad$ Void fraction difference function

$\Gamma_{g} \quad$ Vapor generation rate per volume

$\mu \quad$ Viscosity

$\theta \quad$ Convective quantity

$\rho_{k} \quad$ Density of k-phase

$\sigma \quad$ Surface tension

$\tau_{k} \quad$ Viscous shear stress

$\boldsymbol{\tau}_{k}^{T} \quad$ Turbulent shear stress

$\Omega_{k} \quad$ Inter-phase mass transfer rate per volume

$\Psi_{k} \quad$ Phasic volume flow rate

\section{Subscripts}

\section{$b \quad$ Bubble}

d Entrained liquid (droplets)

$f \quad$ Saturated water or cell face

$g \quad$ Saturated steam or vapor phase

$i \quad$ Interface

$K \quad$ k-field, i.e., continuous liquid, entrained liquid, or vapor

$l \quad$ Continuous liquid

$n \quad$ Noncondensable gases

$s \quad$ Steam or saturated

w Wall

\section{Superscripts}

$m \quad$ Prediction step

$n \quad$ Old time step

$n+1 \quad$ New time step

\section{REFERENCES}

[1] Proc. OECD/CSNI Workshop on Transient ThermalHydraulic and Neutronic Codes Requirements, Annapolis, MD, USA, 5-8 November 1996, NUREG/CP-0159, NEA/CSNI/R(97)4.

[2 ] G. Yadigaroglu, M. Andreani, J. Dreier, and P. Coddinggton, "Trends and needs in experimentation and numerical simulation for LWR safety," Nuclear Engineering and Design, 221, pp. 205-223 (2003).

[ 3 ] D. Bestion, "Extension of CFD codes application to twophase flow safety problems," Nuclear Engineering and Technology, 42, 365-376 (2010).

[ 4 ] G. Yadigaroglu, "Computational fluid dynamics for nuclear applications: From CFD to multi-scale CMFD," Nuclear Engineering and Design, 235(2-4), pp. 153-164 (2005).

[ 5 ] H. Staetke, et al, "Advanced three-dimensional two-phase flow simulation tools for application to reactor safety (ASTAR)," Nuclear Engineering and Design, 235, 379400 (2005).

[6] A. Guelfi, et al., "NEPTUNE: a new software platform for advanced nuclear thermal hydraulics," Nuclear Science and Engineering, 156(3), pp. 281-324 (2007).

[7] D. Bestion, "Status and perspective of two-phase flow modeling in the NEPTUNE multiscale thermal-hydraulic platform for nuclear reactor simulation," Nuclear Engineering and Technology, 37, 511-524 (2005).

[ 8 ] B. Niceno, Y. Sato, A. Badillo, M. Andreani, "Multi-scale modeling and analysis of convective boiling: Towards the prediction of CHF in rod bundles," to be published in the December issue of Nuclear Engineering and Technology (2010).

[9] H. Hosoi, H. Yoshida, "Numerical simulation of air-water two-phase flow in $38 \mathrm{~mm}$ diameter pipe by advanced twofluid model including effects of turbulent diffusion on bubbles," The 7th Korea-Japan Symposium on Nuclear Thermal Hydraulics and Safety (NTHAS7), November 1417, 2010, Chuncheon, Korea.

[10] J.J. Jeong, H.Y. Yoon, I.K. Park, H.K. Cho, and J. Kim, "A Semi-implicit numerical scheme for transient twophase flows on unstructured grids," Nuclear Engineering and Design, 238, pp. 3403-3412 (2008).

[11] Jae Jun Jeong, Han Young Yoon, Ik Kyu Park, Hyoung Kyu Cho, Heedong Lee, "Development and preliminary assessment of a three-dimensional thermal hydraulics code, CUPID," Nuclear Engineering and Technology, 42, 279-296 (2010).

[12] B.J. Yun, D.J. Euh, and C.H. Song, "Investigation of the downcomer boiling phenomena during the reflood phase of a postulated large-break LOCA in the APR1400," Nuclear Technology, 156, pp. 56-68 (2006).

[13] M. Ishii and T. Hibiki, Thermo-Fluid Dynamics of TwoPhase Flow, Springer (2006).

[14] The RELAP5-3D Code Development Team, RELAP5-3D code manual volume I: Code structure, system models and solution methods, Idaho National Engineering and Environmental Laboratory (2001).

[15] J.J. Jeong, K.S. Ha, B.D. Chung, and W.J. Lee, "Development of a multi-dimensional thermal-hydraulic system code, MARS 1.3.1," Annals of Nuclear Energy, 26(18), pp. 1611-1642 (1999).

[16] F. Barre, M. Bernard, "The CATHARE code strategy and assessment," Nuclear Engineenng and Design, 124, 257 284 (1990).

[17] C. Frepoli, J.H. Mahaffy, and K. Ohkawa, "Notes on the implementation of a fully-implicit numerical scheme for a two-phase three-field flow model," Nuclear Engineering and Design, 225, pp. 191-217 (2003).

[18] A. Tentner, et al., "Computational fluid dynamics modeling of two-phase flow in a boiling water reactor fuel assembly," Proc. of Mathematics and Computation, Supercomputing, Reactor Physics and Nuclear and Biological Applications, Avignon, France, 2005.

[19] CD-adapco, "STAR-CD Version 4.0 Methodology Manual", Chapter 13, CD-adapco, UK, 2006.

[20] A. Tentner, et al., "Advances in computational fluid dynamics modeling of two-phase flow in a boiling water reactor fuel assembly," Proc. of ICONE14, Miami, USA, 2006.

[21] A. Tentner, et al., "Computational fluid dynamics modeling of two-phase flow topologies in a boiling water reactor fuel assembly," Proc. of ICONE16, Orlando, USA, 2008.

[22] A. Ioilev, et al., "Advances in the modeling of cladding heat transfer and critical heat flux in boiling water reactor fuel assemblies," Proc. of NURETH-12, Pittsburgh, USA, 2007. 
[23] V. Ustinenko, et al., "Validation of CFD-BWR, a new two-phase computational fluid dynamics model for boiling water reactor analysis," Nuclear Engineering and Design, 238, 660-670 (2008).

[24] K. Yoneda, et al., "Flow structure and bubble characteristics of steam-water two-phase flow in a large-diameter pipe," Nuclear Engineering and Design, 217, 267-281, (2002).

[25] I. Kataoka, M. Ishii, and K. Mishima, "Generation and size distribution of droplet in annular two-phase flow," Trans. ASME, J. Fluid Engineering, 105, 230-238 (1983).

[26] P. Coste et al., "Status of a two-phase CFD approach to the PTS issue," XCFD4NRS Workshop, Grenoble, France, 2008.

[27] D. Drew, L.Y. Cheng, L and R.T. Lahey, "The analysis of virtual mass effect in two-phase flow," Int. J. Multiphase Flow, 5, 233-272 (1979).

[28] A. Tomiyama, H. Tamia, I. Zun, and S. Hosokawa, "Transverse migration of single bubbles in simple shear flows," Chemical Engineering Science, 57, 1849-1858, (2002).

[29] S.P. Antal, R.T. Lahey, J.E. Flaherty, “Analysis of phase distribution in fully developed laminar bubbly two-phase flow," International Journal of Multiphase Flow, 7, 635652, (1991).

[30] R.T. Lahey, M. Lopez de Bertodano and O.C. Jones, "Phase distribution in complex geometry conduits", Nuclear Engineering and Design, 141, 177-201, (1993).

[31] M. Lopez de Bertodano, "Two fluid model for two-phase turbulent jets," Nuclear Engineering and Design, 179, 6574 (1998).

[32] I. Michiyoshi and A. Serizawa, "Turbulence in two-phase bubbly flow," Nuclear Engineering and Design, 95, 253267 (1986).

[33] Y. Sato, M. Sadatomi, K. Sekoguchi, "Momentum and heat transfer in two-phase bubble flow - I", Int. J. Multiphase Flow, 7, 167-177 (1981).

[34] B.J. Yun, A. Splawski, S. Lo, and C.-H. Song, "Prediction of a subcooled boiling flow with mechanistic wall boiling and bubble size models," Proc. CFD4NRS-3, Washington D.C., USA, 14-16 September, 2010.

[35] AEA, CFX-4 Solver Manual, UK, 1997.

[36] R. Cole and W. Rosenhow, "Correlation of bubble departure diameters for boiling of saturated liquids," Chem. Eng. Prog. Symp. Ser. 65 (92), 211-213, 1969.

[37] D.R. Liles and W.H. Reed, "A semi-implicit method for two-phase fluid dynamics," J. Comput. Phys., 26, 390-407 (1978).

[38] H.Y. Yoon and J.J. Jeong, "A continuity based semiimplicit scheme for transient two-phase flows," Journal of Nuclear Science and Technology, 47, No. 9, 779-789 (2010).

[39] I.K. Park, H.K. Cho, H.Y. Yoon, and J.J. Jeong, "Numerical effects of the semi-conservative form of momentum equations for multi-dimensional two-phase flows," Nuclear Engineering and Design, 239, 2365-2371 (2009).

[40] H.K. Cho, H.D. Lee, I.K. Park, J. J. Jeong, "Implementation of a second-order upwind method in a semi-implicit twophase flow code on unstructured meshes," Annals of Nuclear Energy, 37, 606-614 (2010).

[41] Heedong Lee, Jae Jun Jeong, Hyoung Kyu Cho, Han Young Yoon, "An improved numerical scheme to evaluate the pressure gradient on unstructured meshes for two-phase flow analysis," International Communications in Heat and Mass Transfer, 37, 1273-1279 (2010).

[42] N.T. Frink, "Recent progress toward a three-dimensional unstructured Navier-Stokes flow solver," AIAA Paper 940061 (1994).

[43] T.J. Barth and D.C. Jesperson, "The design and application of upwind schemes on unstructured meshes," AIAA Paper 89-0366 (1989).

[44] V. Venkatakrishnan, "Convergence to steady state solutions of the Euler equations on unstructured grids with limiters," Journal of Computational Physics, 118, 120-130 (1995).

[45] J.M. Weiss, "Implicit solution of preconditioned NavierStokes equations using algebraic multigrid," AIAA J. 37, No. 1 (1999).

[46] S.R. Mathur, "A pressure-based method for unstructured meshes," Numerical Heat Transfer, Part B 31, 195-215 (1997).

[47] S.Y. Lee, J.J. Jeong, S.H. Kim, and S.H. Chang, "COBRA /RELAP5; A merged version of the COBRA-TF and RELAP5/MOD3 codes," Nuclear Technology, 99, 177187 (1992).

[48] R. Boer, H. Finnemann, and A. Knoll, "MSLB exercise 2: 3-D kinetics results with RELAP5/PANBOX," Proc. Int. Topl. Mtg. Advances in Reactor Physics and Mathematics and Computation into the Next Millennium (PHYSOR 2000), Pittsburgh, Pennsylvania, May 7-12, 2000, American Nuclear Society.

[49] H.G. Joo, J.J. Jeong, B.O. Cho, W.J. Lee, and S.Q. Zee, "Analysis of the OECD MSLB benchmark problem using the refined core thermal-hydraulic nodalization feature of the MARS/MASTER code," Nuclear Technology, 142, 166-179 (2003).

[50] Jae Jun Jeong, Seung Wook Lee, Jin Young Cho, Bub Dong Chung, and Gyu-Cheon Lee, "A coupled analysis of system thermal-hydraulics and three-dimensional reactor kinetics for a 12-finger control element assembly drop event in a PWR plant," Annals of Nuclear Energy, 37, 1580-1587 (2010).

[51] J.R. Lee, H.K. Cho, J.J. Jeong, "Coupled simulation of component thermal hydraulics and neutron kinetics for a nuclear reactor core with CUPID and MASTER," Transactions of the Korean Nuclear Society Autumn Meeting, Jeju, Korea, October 21-22, 2010.

[52] H.K. Cho, I.K. Park, H.D. Lee, J.J. Jeong, "Constitutive relations and a second-order interpolation scheme for the semi-implicit two-phase flow solver, CUPID," Proceedings of the $17^{\text {th }}$ International Conference on Nuclear Engineering (ICONE17), July 12-16, 2009, Brussels, Belgium.

[53] T. Nagatake, Z. Kawara, and T. Kunugi, "Establishment of experimental data base on dam-breaking problem for validating interface tracking methods," NTHAS6: Sixth Japan-Korea Symposium on Nuclear Thermal Hydraulics and Safety, Okinawa, Japan, Nov. 24-27, 2008.

[54] B.K. Huh, Experimental and Analytical Study of Interfacial Are Transport Phenomena in a Vertical Twophase Flow, Ph. D. Thesis, Seoul National University, Korea (2005).

[55] D.J. Euh, et al., "Transport of local two-phase parameters 
in vertical air/water flow for bubbly and slug flow regime," Transactions of the Korean Nuclear Society Spring Meeting, Pyeongchang, Korea, May 27-28, 2010.

[56] H.K. Cho, B.J. Yun, I.K. Park, and J.J. Jeong, "Computational analysis of downcomer boiling phenomena using a component thermal hydraulic analysis code CUPID," Proc. the $18^{\text {th }}$ International Conference on Nuclear Engineering, Xi'an, China, May 17-21, 2010. 